\title{
PAPER
}

\section{A simplified measurement method of auditory filters for hearing-impaired listeners}

\author{
Takeshi Nakaichi*, Keisuke Watanuki and Shinichi Sakamoto \\ Audiological Engineering Department, RION Co., Ltd., \\ 3-20-41, Higashimotomachi, Kokubunji, 185-8533 Japan
}

(Received 13 December 2002, Accepted for publication 13 May 2003 )

\begin{abstract}
A simplified method for the measurement of auditory filter, in which the filter shape is assumed to be symmetric, is proposed. This method would apply to the clinical diagnosis of hearing impairment and the fitting of hearing aids. The proposed method is based on the notched-noise method and includes procedures for the determination of the masker level and for the estimation of the auditory filter shape from one masked threshold. In order to reduce measuring time, the ascending method is used to detect the signal threshold level in this method. Two experiments were conducted to evaluate this proposed technique: (1) the measurement accuracy of the ascending method was investigated on three normal-hearing subjects and compared with the 2AFC method, and (2) the practicability of this simplified method was evaluated on eleven hearing-impaired and two normalhearing subjects, and compared with the conventional notched-noise method. Results showed that (1) the ascending method has sufficient accuracy for the measurement of the masked threshold and (2) ERB data obtained using the simplified method correlate highly with ERB data obtained using the conventional notched-noise method. It was also shown that each auditory filter could be measured in approximately three minutes.
\end{abstract}

Keywords: Hearing impairment, Auditory filter, Frequency selectivity, Notched noise method, ERB

PACS number: 43.66.Dc, 43.66.Sr, 43.20.Ye

[DOI: $10.1250 /$ ast.24.365]

\section{INTRODUCTION}

The clinical diagnosis of hearing impairment and the fitting of hearing aids require accurate measurement of the auditory indices of hearing-impaired listeners. Several indices are used for measurement; in most cases, audiogram and speech intelligibility are measured. However, because listeners with cochlear hearing losses exhibit complicated auditory characteristics, complete evaluation of auditory characteristics on the basis of existing indices is impossible. New indices and practical measuring methods are therefore required for the successful implementation of advanced auditory prosthesis techniques.

It was reported that frequency selectivity was reduced in many listeners with cochlear hearing loss [1,2]. Tyler $e t$ al. [3] reported that the auditory filter bandwidths of hearing-impaired listeners were often broader than those of normal-hearing listeners, and that frequency selectivity was reduced due to this broadening. Glasberg et al. [4] and Dubno et al. [5] reported that the auditory filter shape also differed for individual hearing-impaired listeners. Moore et

*e-mail: nakaichi@rion.co.jp al. [6], Baer et al. [7] and Nejime et al. [8] attempted to simulate reduced frequency selectivity based on auditory filter shapes, and reported that the simulation of auditory filter shapes could reasonably mimic the effects of reduced frequency selectivity, particularly when applied to the intelligibility of speech in noise. These reports suggested the possibility that auditory filter shape could offer an index for understanding the auditory characteristics of hearing impairments. Auditory filter shape, however, has not previously been investigated in detail from this perspective. This is because the notched-noise method proposed by Patterson et al. [9], which is widely used to measure auditory filters, requires a long measuring time and it is often unsuitable for measuring auditory filters with broadened bandwidth. If it were possible to measure auditory filters for individual hearing-impaired listeners efficiently, the implementation of advanced auditory prosthesis techniques might be significantly improved.

In this paper, a simplified and practical method for measuring auditory filters of individual hearing-impaired listeners is proposed, in which auditory filter shapes are assumed to be symmetric. This method consists of a masker level determination technique for individual hear- 
ing-impaired listeners and an auditory filter shape estimation technique using only one measurement point, which corresponds to one masked threshold in the conventional notched-noise method. In addition, the ascending method on the basis of a method of limits, which detects signal thresholds and can be completed in a short period of time, is used in this simplified method. In Section 2, an overview of the conventional notched-noise method and its controversial aspects for measuring auditory filters of hearingimpaired listeners are described. In Section 3, the simplified measurement method is proposed. In Sections 4 and 5, this proposed method is evaluated in two experiments. In Experiment I, the application of the ascending method for reducing measuring time is examined on three subjects with normal hearing. In Experiment II, auditory filters are measured on eleven hearing-impaired and two normalhearing subjects, using both the proposed simplified method and the conventional notched-noise method. In Section 6, the results and the availability of the simplified measurement method are discussed.

\section{OVERVIEW OF CONVENTIONAL NOTCHED-NOISE METHOD}

\subsection{The Notched-noise Method}

For normal-hearing listeners, the auditory filter shape is asymmetric in frequency [10] and auditory filter bandwidth varies with frequency and with the masker or probe signal level used to measure it [11]. The notched-noise method (hereafter called the conventional method) is widely used to measure auditory filters [9]. Figure 1 shows a schematic

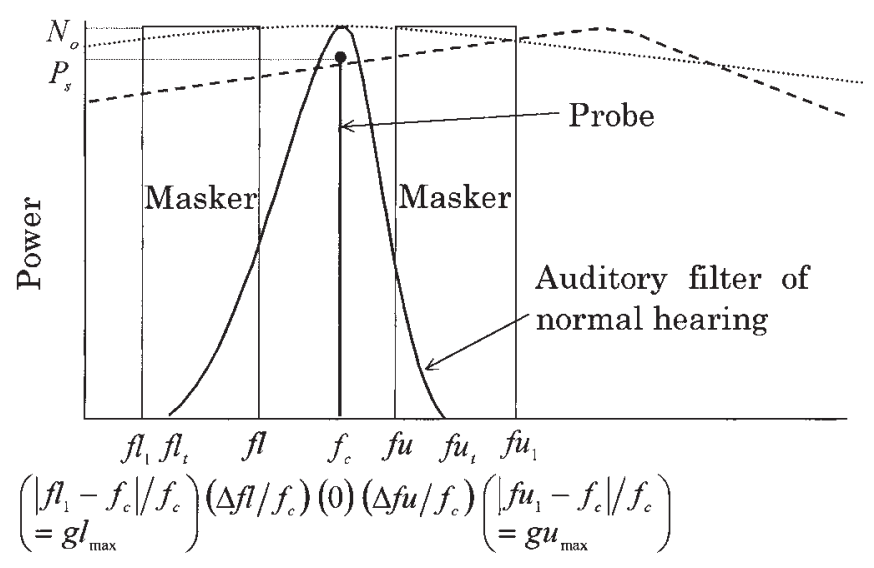

Frequency in $\mathrm{Hz}$

Fig. 1 Schematic diagram of the conventional notchednoise method. Normalized deviations from the center frequency, $f_{\mathrm{c}}$, corresponding to the frequencies, $f l_{1}, f l$, $f_{\mathrm{c}}, f u$ and $f u_{1}$, are shown in parentheses. Solid line denotes the target auditory filter of a normal-hearing listener, dotted line denotes the target auditory filter of a hearing-impaired listener and dashed line denotes an auditory filter of the hearing-impaired listener located in outside regions of the masker. diagram of the conventional method. A sinusoidal probe with frequency, $f_{\mathrm{c}} \mathrm{Hz}$, and a masker with a spectral notch around $f_{\mathrm{c}}$ are used. In most cases, the masker consists of two bands on either side of $f_{\mathrm{c}}$. The notch is placed both symmetrically and asymmetrically about $f_{\mathrm{c}}$ in order to estimate the asymmetry of the auditory filter shape $[12,13]$. These stimuli are presented simultaneously to the subject, and then the masked thresholds are measured as a function of the notch width. Auditory filter shapes are derived from the masked thresholds, based on the assumption of the power-spectrum model of masking [10,14]. The power of the probe signal at the masked threshold is given by:

$$
P_{\mathrm{s}}=K \int_{0}^{\infty} N(f) W(f) d f
$$

where $f$ is the frequency, and $N(f)$ represents the longterm power spectrum of the masker. $W(f)$ is the weighting function, which describes the shape of the auditory filter, and $K$ is a constant, which is related to the efficiency of the detection mechanism following the auditory filter [14]. If the auditory filter was assumed to have the form of the roex $(p, r)$ filter [15], then

$$
W(g)=(1-r)(1+p g) \exp (-p g)+r
$$

where $g \quad\left(=\left|f-f_{\mathrm{c}}\right| / f_{\mathrm{c}}=\Delta f / f_{\mathrm{c}}\right)$ is the normalized deviation from the center frequency $\left(f_{\mathrm{c}}\right), p$ denotes the pass band and $r$ denotes the dynamic range of the filter. By applying Eq. (2) to Eq. (1), it can be shown that

$$
\begin{aligned}
P_{\mathrm{s}}= & K f_{\mathrm{c}} N_{\mathrm{o}}\left(\int_{\Delta f l / f_{\mathrm{c}}}^{g l_{\max }} W(g) d g+\int_{\Delta f u / f_{\mathrm{c}}}^{g u_{\max }} W(g) d g\right) \\
= & K f_{\mathrm{c}} N_{\mathrm{o}}\left[\left[\frac{-(1-r)(2+p g)}{p e^{p g}}+r g\right]_{\Delta f l / f_{\mathrm{c}}}^{g l_{\max }}\right. \\
& \left.+\left[\frac{-(1-r)(2+p g)}{p e^{p g}}+r g\right]_{\Delta f u / f_{\mathrm{c}}}^{g u_{\max }}\right]
\end{aligned}
$$

where the first term inside the parentheses in Eq. (3) shows the integral for the lower side of the masker and the second term shows the upper side. The masker level has uniform power spectrum, $N_{\mathrm{o}}$, and the width of the notch ranges from $f l$ to $f u$. The normalized deviations from the center frequency, $f_{\mathrm{c}}$, to the inside edges of the masker are $\Delta f l / f_{\mathrm{c}}$ $\left(=\left|f l-f_{\mathrm{c}}\right| / f_{\mathrm{c}}\right)$ and $\Delta f u / f_{\mathrm{c}}\left(=\left|f u-f_{\mathrm{c}}\right| / f_{\mathrm{c}}\right) \cdot g l_{\max }$ is the lower limit and $g u_{\max }$ is the upper limit. In the conventional method, these limits are often superimposed to the outside edges of the masker (i.e., $g l_{\max }=\left|f l_{1}-f_{\mathrm{c}}\right| / f_{\mathrm{c}}$ and $\left.g u_{\max }=\left|f u_{1}-f_{\mathrm{c}}\right| / f_{\mathrm{c}}\right)$. The value of $p$ is allowed to differ for the upper and lower halves of the auditory filter. The auditory filter shape can be estimated by finding the values of $p, r$ and $K$ that give the best fit to the masked thresholds [16]. The correction for the non-flat transmission characteristics of the outer and middle ear is often made in this 
fitting procedure [16,17]. Glasberg et al. [18] reported that reasonable correction could be made further by applying a stimulus that had a constant level at the eardrum.

\subsection{Controversial Aspects of the Conventional Meth- od When Measuring Auditory Filters of Hearing- impaired Listeners}

2.2.1. Measuring time

Considering that most hearing-impaired listeners are elderly, it is required that the measurement for an auditory index is completed within 30-40 minutes for the clinical diagnosis of hearing impairment and the fitting of hearing aids. When auditory filters are measured using the conventional method, it is predicted to drastically exceed the measuring time of 30-40 minutes for the following three reasons. First, the technique of determining the masked thresholds requires a long measuring time. In the conventional method, the 2AFC (two alternative forced choice) method [19] is most often used to collect masked thresholds, from which it is possible to collect statistically acceptable data. The $2 \mathrm{AFC}$ method, however, required the measuring time of approximately 30 minutes to collect each masked threshold when the filter is measured under the same conditions as reported by Patterson et al. [15], as described in Section 4.2. Second, between thirteen and nineteen masked thresholds are required to estimate an asymmetric auditory filter shape $[12,13,18]$. If the auditory filter shape is assumed to be symmetric, five or more masked thresholds are still required [5,15]. Although, Stone et al. [20] reported that it was possible to estimate an asymmetric auditory filter shape using only five masked thresholds, completion of the measurement still took 150 minutes (30 minutes $\times$ five masked thresholds). Third, auditory filter bandwidths of hearing-impaired listeners can be predicted to vary abruptly with frequency and masker level. Therefore, twelve or more auditory filters, which are measured with three probe levels (e.g., 10, 20 and $30 \mathrm{~dB}$ above the hearing threshold) under four frequencies (e.g., $250,500,1,000$ and $2,000 \mathrm{~Hz}$ ), may be required to obtain the auditory characteristics of the hearing-impaired listeners. These measurements require several days, even if Stone's method is used.

If the ascending method is used instead of the 2AFC method, the measuring time for each auditory filter can be reduced. Since the ascending method can collect the masked threshold in approximately 2 minutes, the measuring time is reduced to approximately 10 minutes (i.e., 2 minutes $\times$ five masked thresholds). Even so, the measuring time for these twelve filters mentioned above is still two hours. Reducing the number of measuring points is needed to implement a practical measurement method of auditory filters.

\subsubsection{Determination technique of the masker level}

In many cases, a masker level between 40 and $50 \mathrm{~dB}$ $\mathrm{SPL} / \mathrm{Hz}$ is used to measure the auditory filters of normalhearing listeners [9,12]. For hearing-impaired listeners, however, the masker at the same level as that for the normal-hearing listener may not be perceived due to the hearing threshold elevation. Even if it is possible to measure the auditory filter using a masker at a specified level, the difference of the masked threshold at $\Delta f / f_{\mathrm{c}}=0$ (i.e., the masker has no notch) and the hearing threshold (hereafter called "the dynamic range of the masked thresholds") would differ according to the level of hearing threshold elevation for individual listeners. The estimated dynamic range of the auditory filter may also be different for individual listeners, since the dynamic range of the auditory filter is approximately equal to that of the masked thresholds [12]. It is difficult to compare and evaluate auditory filter shapes in such a situation.

\subsubsection{Measurement range}

By measuring masked thresholds in the range of $\Delta f / f_{\mathrm{c}}=0.0 \sim 0.6$, it is possible to estimate accurate auditory filter shapes of normal-hearing listeners. For hearing-impaired listeners, however, measuring in the measurement range for normal-hearing listeners may not provide a sufficient estimation. To estimate auditory filter shapes with broadened bandwidth accurately, masked thresholds using the masker with a larger notch width must be measured. It is difficult, however, to determine the sufficient measurement range for hearing-impaired individuals before the measurement.

\subsubsection{Bandwidth of masker}

\subsubsection{Bandwidth of masker}

For hearing-impaired listeners, the hearing thresholds may vary abruptly with frequency changes, and the auditory filter bandwidths may also vary abruptly with changes in probe frequency and/or masker level. Therefore, it should be noted that the auditory filters located in the outside regions (the dashed line in Fig. 1) may affect the measurement of the target filter (the dotted line in Fig. 1). To prevent such an effect, a white noise, ranging from $0 \mathrm{~Hz}$ to the Nyquest frequency, to which a spectral notch is applied (hereafter called the white-noise masker with a spectral notch), should be used as the masker for the measurement.

2.2.4.2. The limit of the integral to estimate the filter shape

In most previous reports, the limits of the integral were superimposed to the outside edges of the masker, since the masker consists of two bands on either side of $f_{\mathrm{c}}$ (i.e., $g l_{\max }=\left|f l_{1}-f_{\mathrm{c}}\right| / f_{\mathrm{c}}$ and $\left.g u_{\max }=\left|f u_{1}-f_{\mathrm{c}}\right| / f_{\mathrm{c}}\right)$. When the white-noise masker with a spectral notch is used for the measurement, $g l_{\max }$ and $g u_{\max }$ are 1.0 and $\left|f_{\mathrm{n}}-f_{\mathrm{c}}\right| / f_{\mathrm{c}}$ respectively, where $f_{\mathrm{n}}$ is the Nyquest frequency of the masker. On the other hand, as shown in Fig. 1, if the target 
auditory filter attenuates completely at $f l_{\mathrm{t}}$ and $f u_{\mathrm{t}}$, components of the masker ranging from $f l_{1}$ to $f l_{\mathrm{t}}$ and from $f u_{\mathrm{t}}$ to $f u_{1}$ would not affect the computational result of the integrals. Therefore, it may be allowed to define $g l_{\max }$ and $g u_{\max }$ as $\left|f l_{\mathrm{t}}-f_{\mathrm{c}}\right| / f_{\mathrm{c}}$ and $\left|f u_{\mathrm{t}}-f_{\mathrm{c}}\right| / f_{\mathrm{c}}$, respectively. Patterson et al. [15] measured the auditory filters of normal-hearing listeners using a white-noise masker with a spectral notch at a level of $40 \mathrm{~dB} \mathrm{SPL} / \mathrm{Hz}$. In this measurement, the masker with a symmetric notch around $f_{\mathrm{c}}$ was used (i.e., $\Delta f=\Delta f l=\Delta f u$ ), since the filter shape was assumed to be symmetric. The roex $(p, r)$ filter was used as the $W(g)$. They set $g l_{\max }$ and $g u_{\max }$ at 0.8 concurrently instead of at 1.0 and $\left|f_{\mathrm{n}}-f_{\mathrm{c}}\right| / f_{\mathrm{c}}$ respectively. Figure 2(a) shows the masked thresholds and the fitted lines at $f_{\mathrm{c}}=250 \mathrm{~Hz}$ for a listener with normal hearing; this measurement is based on the conditions reported by Patterson et al. [9,15], and Fig. 2(b) shows the auditory filter shape estimated. The white-noise masker $\left(f_{\mathrm{n}}=11,025 \mathrm{~Hz}\right)$ with a spectral notch was used. Solid lines denote the results with $g l_{\max }=1.0$ and $g u_{\max }=$ $\left|f_{\mathrm{n}}-f_{\mathrm{c}}\right| / f_{\mathrm{c}}$ and dashed lines denote the results with $g l_{\max }=g u_{\max }=0.8$. Although no obvious difference in these two conditions is observed in the fitting results of the masked thresholds, the dynamic range of the filter estimated with $g l_{\max }=1.0$ and $g u_{\max }=\left|f_{\mathrm{n}}-f_{\mathrm{c}}\right| / f_{\mathrm{c}}$ is larger than that with $g l_{\max }=g u_{\max }=0.8$. The estimation with $g l_{\max }=g u_{\max }=0.8$ is more acceptable than that with $g l_{\max }=1.0$ and $g u_{\max }=\left|f_{\mathrm{n}}-f_{\mathrm{c}}\right| / f_{\mathrm{c}}$ since the dynamic range of the filter with $g l_{\max }=g u_{\max }=0.8$ is approx-
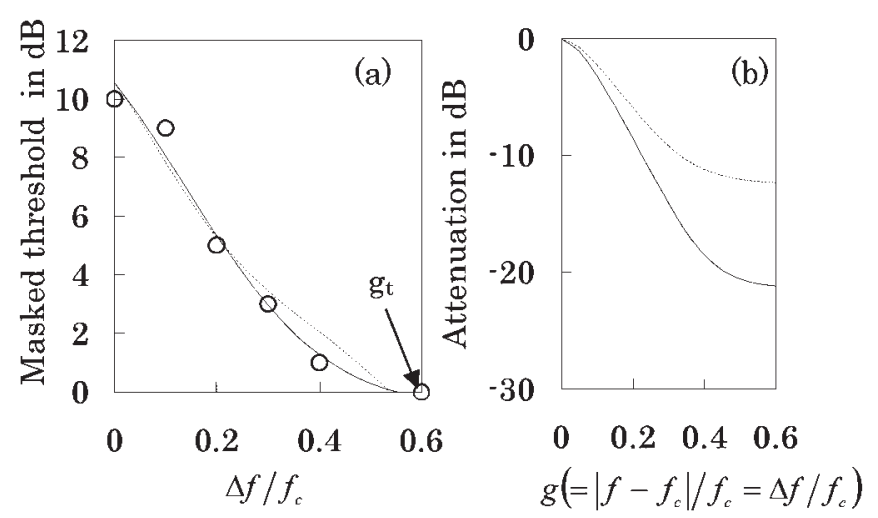

Fig. 2 Masked thresholds, fitted lines and auditory filter shapes at $250 \mathrm{~Hz}$. (a) The masked thresholds and fitted lines. The abscissa denotes notch width $\Delta f / f_{\mathrm{c}}$ and the ordinate denotes masked threshold level in $\mathrm{dB}$ when the hearing threshold is set at $0 \mathrm{~dB}$. The lines are fitted by the roex $(p, r)$ model. Solid lines denote the results with $g l_{\max }=1.0$ and $g u_{\max }=\left|f_{\mathrm{n}}-f_{\mathrm{c}}\right| / f_{\mathrm{c}}$ and dashed lines denote the results with $g l_{\max }=g u_{\max }=0.8 . g_{\mathrm{t}}$ denotes the notch width at which the masked threshold just approaches the hearing threshold. (b) The estimated auditory filter shapes. The abscissa denotes $g$ $\left(=\left|f-f_{\mathrm{c}}\right| / f_{\mathrm{c}}=\Delta f / f_{\mathrm{c}}\right)$ and the ordinate denotes attenuation of the filter. imately $10 \mathrm{~dB}$, which is approximately equal to the dynamic range of the masked thresholds. The difference in these results is due to the fact that the roex $(p, r)$ filter is an asymptotic function (i.e., this function value can never be zero). This means that the integral value in the range of $\Delta f / f_{\mathrm{c}} \geqq 0.8$ is not zero and so it must contribute to the calculation of the integral. Thus, $g l_{\max }$ and $g u_{\max }$ should be set at suitable values, when the roex $(p, r)$ filter is used as the $W(f)$ function and the white-noise with a spectral notch is used as the masker. Additionally, the auditory filter bandwidth varies with the masker or probe signal level used to measure it (i.e., the bandwidth varies with the dynamic range) [11]. The values of $g l_{\max }$ and $g u_{\max }$, therefore, should be set according to the auditory filter dynamic range and the bandwidth. Furthermore, the values of $g l_{\max }$ and $g u_{\max }$ for hearing-impaired listeners should be set on an individual basis, since their auditory filter bandwidths may be broadened and the degree of the broadening would differ from one individual to another.

\section{SIMPLIFIED MEASUREMENT METHOD OF AUDITORY FILTERS}

It is well-known that auditory filter shape is asymmetric, as described in Section 2.1. However, the goal of this study is to introduce individual auditory filters as a new auditory index for improving the quality of existing fitting procedures of hearing aids and for developing advanced auditory prosthesis techniques. Considering this goal, implementing a practical method is an urgent priority. Taking count of reducing measuring time, therefore, this proposed method is discussed in this study on the assumption that auditory filter shape is symmetric.

\subsection{Procedure of the Simplified Method}

This procedure consists of both a masker level determination technique and an auditory filter shape estimation technique. The masker level determination technique can determine the dynamic range of the auditory filter, and the auditory filter shape estimation technique uses only one measurement point corresponding to one masked threshold. Figure 3 shows a schematic diagram of the proposed measurement procedure.

Firstly, the level of a sinusoidal probe at $f_{\mathrm{c}} \mathrm{Hz}$ is fixed at $T+x \mathrm{~dB}$ SPL, where $T$ is the hearing threshold of the subject and $x(>0)$ is an arbitrary value. The probe and a white-noise masker without a spectral notch, at a level that does not mask the probe, are presented simultaneously to the subject, and then the masker level is gradually increased. The masker level needed to just mask the probe is defined as $n_{x} \mathrm{~dB}$ SPL/Hz. When masked thresholds are measured using the conventional method with the observed masker level $n_{x}$, the dynamic range of the masked thresholds will be approximately $x \mathrm{~dB}$. Since it can be 


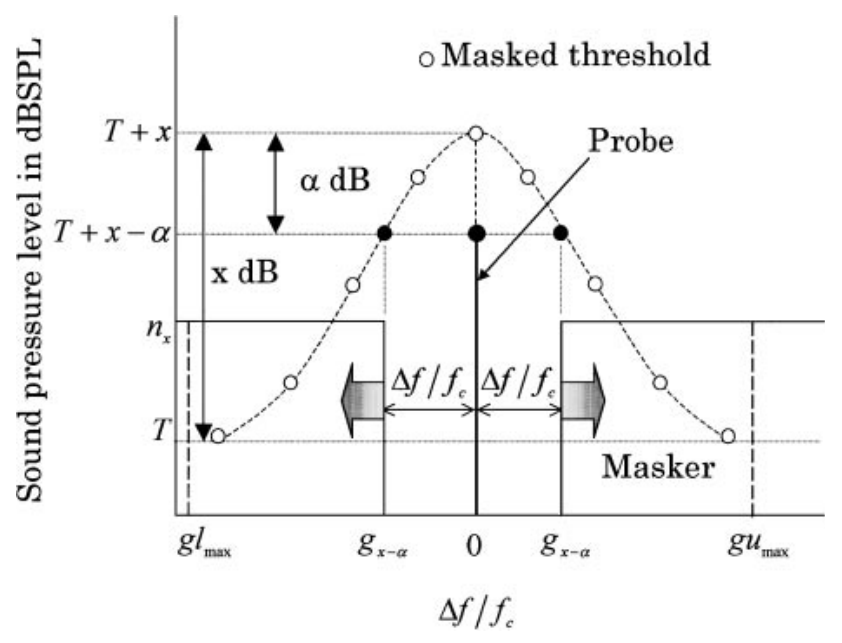

Fig. 3 Schematic diagram of the simplified method.

assumed that the dynamic range of the auditory filter is approximately equal to that of the masked thresholds [12], the dynamic range of the auditory filter can be also assumed to be $x \mathrm{~dB}$.

Secondly, the probe level is fixed at $T+x-\alpha \mathrm{dB}$ SPL, where $\alpha(x>\alpha>0)$ is an arbitrary value. The masker level is fixed at $n_{x}$. These stimuli are presented simultaneously to the subject. Since the masker is a white noise (i.e., $\Delta f / f_{\mathrm{c}}=0$ ), the subject cannot detect the probe. A small spectral notch centered at $f_{\mathrm{c}} \mathrm{Hz}$ is applied to the masker, and then the notch width $\Delta f / f_{\mathrm{c}}$ is gradually increased. The notch width needed to just detect the probe is defined as $g_{x-\alpha}$, corresponding to the one masked threshold in the conventional method.

\subsection{Derivation of Auditory Filter Shape}

In this simplified method, the auditory filter was assumed to have the form of the roex $(p, r)$ filter [15]. Since the dynamic range of the auditory filter is fixed at $x$ $\mathrm{dB}$, as described in Section 3.1, the parameter $r$ can be assumed to be $10 \log _{10} r=-x$. Therefore, the auditory filter shape can be estimated by determining the value of $p$ only.

The difference of $P_{\mathrm{S}}(0)$ and $P_{\mathrm{s}}\left(g_{x-\alpha}\right)$ is $\alpha \mathrm{dB}$ as shown in Fig. 3, then $\alpha$ is given by:

$$
\alpha=10 \log _{10}\left\{P_{\mathrm{s}}(0) / P_{\mathrm{s}}\left(g_{x-\alpha}\right)\right\}
$$

By applying Eqs. (2) and (3) to Eq. (4), it can be shown that

$$
\begin{aligned}
\alpha=10 \log _{10}\left\{\frac{K f_{\mathrm{c}} N_{\mathrm{o}}\left(\int_{0}^{g l_{\max }} W(g) d g+\int_{0}^{g u_{\max }} W(g) d g\right)}{K f_{\mathrm{c}} N_{\mathrm{o}}\left(\int_{g_{x-\alpha}}^{g l_{\max }} W(g) d g+\int_{g_{x-\alpha}}^{g u_{\max }} W(g) d g\right)}\right\} \\
=10 \log _{10}\left[\frac{\left[\frac{-(1-r)(2+p g)}{p e^{p g}}+r g\right]_{0}^{g l_{\max }}+\left[\frac{-(1-r)(2+p g)}{p e^{p g}}+r g\right]_{0}^{g u_{\max }}}{\left[\frac{-(1-r)(2+p g)}{p e^{p g}}+r g\right]_{g_{x-\alpha}}^{g l_{\max }}+\left[\frac{-(1-r)(2+p g)}{p e^{p g}}+r g\right]_{g_{x-\alpha}}^{g u_{\max }}}\right]
\end{aligned}
$$

where the parameter $r$ can be set at $10 \log _{10} r=-x \mathrm{~dB}, \alpha$ is an arbitrary value, which is set by the experimenter, and $g_{x-\alpha}$ is the measured bandwidth. If the values of $g l_{\max }$ and $g u_{\max }$ are determined, the only unknown value is $p$, which can be derived.

\subsection{Determination of the Integral Range}

In this section, we implement a technique, which uses the $g_{x-\alpha}$ measured in the simplified method, to estimate the acceptable $g l_{\max }$ and $g u_{\max }$ values for individual hearing impaired listeners. These values should be determined according to the auditory filter dynamic range and the bandwidth, as described in subsection 2.2.4 of Section 2.2. Since $g_{x-\alpha}$ in the simplified method is a bandwidth of the auditory filter measured according to the dynamic range ( $x$ $\mathrm{dB}$ ), we assume that $g l_{\max }$ and $g u_{\max }$ relate to $g_{x-\alpha}$ proportionally, i.e.,

$$
\begin{aligned}
g l_{\max } & = \begin{cases}1.0 & \text { for } A_{x-\alpha} g_{x-\alpha}>1.0 \\
A_{x-\alpha} g_{x-\alpha} & \text { for } A_{x-\alpha} g_{x-\alpha} \leq 1.0\end{cases} \\
g u_{\max } & = \begin{cases}\left|f_{\mathrm{n}}-f_{\mathrm{c}}\right| / f_{\mathrm{c}} & \text { for } A_{x-\alpha} g_{x-\alpha}>\left|f_{\mathrm{n}}-f_{\mathrm{c}}\right| / f_{\mathrm{c}} \\
A_{x-\alpha} g_{x-\alpha} & \text { for } A_{x-\alpha} g_{x-\alpha} \leq\left|f_{\mathrm{n}}-f_{\mathrm{c}}\right| / f_{\mathrm{c}}\end{cases}
\end{aligned}
$$

where $A_{x-\alpha}$ is a parameter, which is set for each dynamic range $x$ and an arbitrary value $\alpha$. In this study, the $A_{x-\alpha}$ values are determined from several masked thresholds measured in previous studies as well as the experimental results for the two normal-hearing subjects described in Section 5, on the basis of following assumptions.

Acceptable values of $g l_{\max }$ and $g u_{\max }$ for normalhearing listeners are regarded as 0.8 , based on Patterson et al. [15], when the auditory filter shape is assumed to be symmetric and the masker level is fixed between 40 and $50 \mathrm{~dB} \mathrm{SPL} / \mathrm{Hz}$. On the other hand, acceptable values of $g l_{\max }$ and $g u_{\max }$ have to be found for each masker level as 
described in subsection 2.2.4 of Section 2.2. However, there are not many reports in which the auditory filter is measured using the masker at the level of outside range between 40 and $50 \mathrm{~dB}$ SPL/Hz, and there are no reports in which the acceptable values of $g l_{\max }$ and $g u_{\max }$ are investigated for such masker levels. For estimating these acceptable values, we define the notch width at which the masked threshold just approaches the hearing threshold as $g_{\mathrm{t}}$ shown in Fig. 2(a), and assume that the acceptable values of $g l_{\max }$ and $g u_{\max }$ relate to the value of $g_{t}$ proportionally, i.e.,

$$
\begin{aligned}
g l_{\max } & = \begin{cases}1.0 & \text { for } B g_{\mathrm{t}}>1.0 \\
B g_{\mathrm{t}} & \text { for } B g_{\mathrm{t}} \leq 1.0\end{cases} \\
g u_{\max } & = \begin{cases}\left|f_{\mathrm{n}}-f_{\mathrm{c}}\right| / f_{\mathrm{c}} & \text { for } B g_{\mathrm{t}}>\left|f_{\mathrm{n}}-f_{\mathrm{c}}\right| / f_{\mathrm{c}} \\
B g_{\mathrm{t}} & \text { for } B g_{\mathrm{t}} \leq\left|f_{\mathrm{n}}-f_{\mathrm{c}}\right| / f_{\mathrm{c}}\end{cases}
\end{aligned}
$$

Observing the masked thresholds measured by Glasberg and Moore [4,12], and Shailer et al. [21], all of whom used a masker with a symmetric notch around $f_{\mathrm{c}}$ and a level between 40 and $50 \mathrm{~dB} \mathrm{SPL} / \mathrm{Hz}$, the values of $g_{\mathrm{t}}$ seem to be between 0.6 and 0.7 for normal-hearing listeners. Thus, the value of $B$ can be predicted to be approximately 1.2 , which is derived from Eqs. (8) and (9) with $g_{\mathrm{t}} \cong 0.6-0.7$ and $g l_{\max }=g u_{\max }=0.8$. If the value of $g_{\mathrm{t}}$ can be found, acceptable values of $g l_{\max }$ and $g u_{\max }$ can be estimated when using the masker at the outside level between 40 and $50 \mathrm{~dB}$ $\mathrm{SPL} / \mathrm{Hz}$, by using Eqs. (8) and (9), and this predicted value, $B=1.2$. The $A_{x-\alpha}$ values in Eqs. (6) and (7) can be calculated by using both the acceptable values of $g l_{\max }$ and $g u_{\max }$, estimated from the assumptions described above, and the value of $g_{x-\alpha}$ measured for normal-hearing subjects in the simplified method. Figure 4 shows the procedure for determining the $A_{x-\alpha}$ values in this paper. The $A_{x-\alpha}$ values are determined experimentally for two normal-hearing subjects.

\section{EXPERIMENT I (EVALUATION OF THE ASCENDING METHOD)}

As described in Section 3, the auditory filter shape can be derived from only one measuring point, $g_{x-\alpha}$, using this simplified method. To reduce the measuring time further, we attempted to use the ascending method to collect the signal thresholds $\left(T, n_{x}\right.$ and $\left.g_{x-\alpha}\right)$ in the simplified method. In Experiment I, auditory filters were measured with the conventional method using the ascending method and the 2AFC method at a probe frequency of $1,000 \mathrm{~Hz}$. The filter shapes, which were assumed to have the form of the roex $(p, r)$, were estimated, then the accuracy and the measuring time of the ascending method were evaluated.

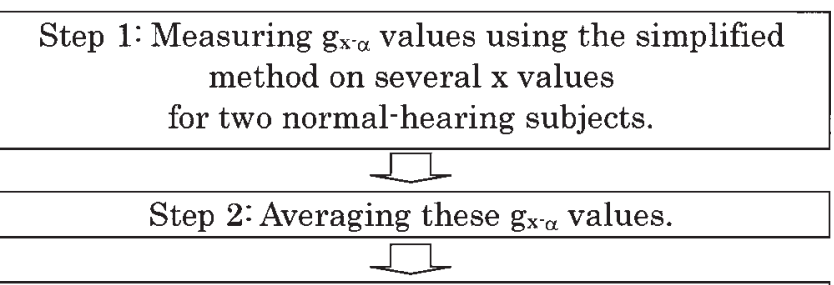

Step 3: Measuring masked thresholds for finding $g_{t}$ values on several $x$ values using the conventional method.

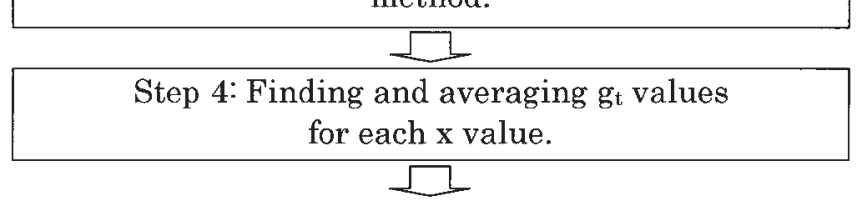

Step 5: Calculating acceptable $g \mathrm{l}_{\max }$ and $g u_{\max }$ values for each $\mathrm{x}$ and $\alpha$ value based on the assumption of Eqs. (8), (9) and the predicted value, $\mathrm{B}=1.2$.

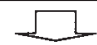

Step 6: Calculating $\mathrm{A}_{\mathbf{x}^{\prime} \cdot \alpha}$ using these $\mathrm{gl}_{\max }, \mathrm{gu}_{\max }$ in Step 5 and $g_{x^{-\alpha}}$ in Step 2 .

$\mathrm{A}_{\mathrm{x}-\alpha}=\mathrm{gl}_{\max } / \mathrm{g}_{\mathrm{x}^{-} \alpha}=\mathrm{gu}_{\max } / \mathrm{g}_{\mathrm{x}^{-} \alpha}$

Fig. 4 Procedure for determining $A_{x-\alpha}$ values.

\subsection{Method}

\subsubsection{Subjects}

Three normal-hearing subjects participated in this experiment. Their ages ranged from 26 to 28 years and their hearing thresholds were less than $10 \mathrm{~dB} \mathrm{HL}$ at $1,000 \mathrm{~Hz}$.

\subsubsection{Stimuli and equipment}

A white-noise masker with a spectral notch was used. The masker level was fixed at $40 \mathrm{~dB} \mathrm{SPL} / \mathrm{Hz}$ in both methods, then the threshold for detecting a probe was measured. The notch was placed symmetrically at $f_{\mathrm{c}}$, and the values of $\Delta f / f_{\mathrm{c}}$ were $0.0,0.1,0.2,0.3,0.4$, and 0.6 . In the ascending method, the masker was presented continuously. The probe had a steady-state portion of $400 \mathrm{~ms}$ and rise/fall portions shaped with $50-\mathrm{ms}$ cosine ramps. The interval between probes was $400 \mathrm{~ms}$. In the $2 \mathrm{AFC}$ method, the masker had the same time contour as the probe in the ascending method. The probe had a steady-state portion of $300 \mathrm{~ms}$ and rise/fall portions shaped with 50-ms cosine ramps, which were presented with a 50-ms delay from the masker. The interval between maskers was $500 \mathrm{~ms}$. The probe and the masker were reproduced using a stereo sound card (Sound Blaster, X-Gamer) in a personal computer. The probe level and the masker level were adjusted independently by each analog attenuator (KIKUSUI, 984 A) and mixed (VICTOR, MI-5000). This mixed signal was transmitted to an audiometer (RION, AA-73) and presented to the subject via supra-aural earphones (RION, AD-02). The subject responded using an answering button in the ascending method and using a computer keyboard in the 
2AFC method. The LED, which signifies the start of each trial and the presence of test signals, was used only in the 2AFC method. All stimuli were produced digitally on an engineering workstation (Fujitsu, GP400S Model 5), with a sampling frequency of $22,050 \mathrm{~Hz}$ and 16 bits. The spectral notch of the masker was produced using a FIR filter with 8,192 taps.

\subsubsection{Procedure}

In the ascending method, the step size of the probe level was $2 \mathrm{~dB}$. This measurement was repeated three times on different days and averaged. In the 2AFC method, test signals were presented in pairs. The probe occurred in only one of the two test signals. Subjects were instructed to indicate when the probe occurred in a test signal. The probe level at the start of the test was $5 \mathrm{~dB}$ above the hearing threshold level of the subject. The probe level was decreased after two consecutive correct responses or increased for each incorrect response. The step size of the probe level was initially set at $8 \mathrm{~dB}$. The step size was changed to $2 \mathrm{~dB}$ after six turnarounds (i.e., after six alternating correct and incorrect responses), and then eighty paired signals were presented. Levels at the turnaround, excluding the first six, were averaged and recorded. When the standard deviation of these levels was greater than $3.5 \mathrm{~dB}$, the measured value was removed from the recorded data. This measurement was repeated until two measured values with a standard deviation less than $3.5 \mathrm{~dB}$ were obtained. Furthermore, when the difference between the two measured values was greater than $5 \mathrm{~dB}$, a third value was also measured. The average of these two or three measured values represents the masked threshold.

\subsection{Results}

Table 1 shows the parameter values, ERB (equivalent rectangular bandwidths) [16], the root-mean-squared (rms) error values and the standard deviations for each subject. Filter shape estimates were made using the L-M (Levenberg-Marquardt) method [22], in which $g l_{\max }$ and $g u_{\max }$ were set at 0.8 . The ERB value is calculated by

$$
E R B=f_{\mathrm{c}}\left(\int_{0}^{g l_{\max }} W(g) d g+\int_{0}^{g u_{\max }} W(g) d g\right) / W(0)
$$

As shown in Table 1, there is no obvious difference between the auditory filters identified with the ascending method and the 2AFC method. The rms value for the ascending method is greater than that for the 2AFC method. The value for the ascending method, however, is also acceptable for the estimation. In addition, the measurement error of the ascending method is not at all large in comparison with the $2 \mathrm{AFC}$ method, since the standard deviations of the measured data recorded using the ascending method are smaller than those recorded using the $2 \mathrm{AFC}$ method. These results show that the ascending method is applicable for measuring auditory filters in the conventional method. The measuring time for each masked threshold needed was approximately 2 minutes for the ascending method and approximately 30 minutes for the 2AFC method.

\section{EXPERIMENT II (EVALUATION OF THE SIMPLIFIED METHOD)}

Auditory filters were measured by both the simplified method and the conventional method, in which, the ascending method was used to detect the signal thresholds. The results were compared and the practicability of the simplified method was evaluated.

\subsection{Method}

\subsubsection{Subjects}

Eleven hearing-impaired subjects and two normalhearing subjects participated in this experiment. Their ages ranged from 20 to 88 years. The group consisted of eight females and five males. Table 2 shows the individual profile and hearing thresholds for the hearing-impaired subjects. Hearing thresholds were measured with a step size of $2 \mathrm{~dB}$. For the normal-hearing subjects, hearing thresholds were less than $18 \mathrm{~dB}$ HL for all frequencies.

\subsubsection{Stimuli and equipment}

Stimuli and equipment were identical to those used for the ascending method in Experiment I. A RION AA-75 audiometer and RION AD-06 supra-aural earphones were used in this experiment.

\subsubsection{Procedure}

The probe signals had frequencies of $250,500,1,000$, 2,000 and $4,000 \mathrm{~Hz}$. The notch was placed symmetrically about the probe frequency. In the simplified method, the

Table 1 Parameter values and ERB obtained from the ascending method and the 2AFC method.

\begin{tabular}{|c|c|c|c|c|c|c|c|c|c|c|c|c|}
\hline \multirow[b]{2}{*}{ Sub. } & \multicolumn{6}{|c|}{ The ascending method } & \multicolumn{6}{|c|}{ The 2AFC method } \\
\hline & $p$ & $\begin{array}{c}r \\
(\mathrm{~dB})\end{array}$ & $\begin{array}{c}K \\
(\mathrm{~dB})\end{array}$ & $\begin{array}{l}E R B \\
(\mathrm{~Hz})\end{array}$ & $\begin{array}{l}r m s \\
\text { (dB) }\end{array}$ & $\begin{array}{l}\text { s.d. } \\
\text { (dB) }\end{array}$ & $p$ & $\begin{array}{c}r \\
(\mathrm{~dB})\end{array}$ & $\begin{array}{c}K \\
(\mathrm{~dB})\end{array}$ & $\begin{array}{l}E R B \\
(\mathrm{~Hz})\end{array}$ & $\begin{array}{l}r m s \\
(\mathrm{~dB})\end{array}$ & $\begin{array}{l}\text { s.d. } \\
\text { (dB) }\end{array}$ \\
\hline A & 27.6 & -44.5 & -2.4 & 145 & 0.39 & 1.2 & 27.1 & -49.3 & -6.5 & 148 & 0.07 & 3.0 \\
\hline B & 25.2 & -50.5 & -2.9 & 158 & 0.33 & 2.3 & 25.4 & -44.0 & -5.2 & 157 & 0.04 & 2.7 \\
\hline $\mathrm{C}$ & 24.9 & -45.4 & -1.4 & 161 & 0.18 & 1.6 & 28.3 & -46.6 & -1.7 & 142 & 0.09 & 2.8 \\
\hline
\end{tabular}

s.d.: standard deviation 
Table 2 Profiles and hearing thresholds for tested ears of individual subjects.

\begin{tabular}{|c|c|c|c|c|c|c|c|c|}
\hline \multirow[b]{2}{*}{ Sub. } & \multirow[b]{2}{*}{ Age } & \multirow[b]{2}{*}{ Sex } & \multirow[b]{2}{*}{ Ear } & \multicolumn{5}{|c|}{ Frequency $(\mathrm{Hz})$} \\
\hline & & & & 250 & 500 & $\begin{array}{l}1,000 \\
(\mathrm{~dB} H L)\end{array}$ & 2,000 & 4,000 \\
\hline $\mathrm{D}$ & 79 & M & $\mathrm{R}$ & 19 & 40 & 46 & 42 & 44 \\
\hline E & 77 & M & $\mathrm{R}$ & 37 & 36 & 45 & 54 & 72 \\
\hline $\mathrm{F}$ & 88 & $\mathrm{~F}$ & $\mathrm{~L}$ & 59 & 66 & 64 & 46 & 46 \\
\hline G & 77 & $\mathrm{~F}$ & $\mathrm{~L}$ & 25 & 38 & 52 & 58 & 58 \\
\hline $\mathrm{H}$ & 62 & $\mathrm{~F}$ & $\mathrm{~L}$ & 71 & 76 & 70 & 66 & 76 \\
\hline I & 66 & $\mathrm{~F}$ & $\mathrm{R}$ & 39 & 44 & 52 & 54 & 62 \\
\hline $\mathrm{J}$ & 82 & $\mathrm{~F}$ & $\mathrm{R}$ & 11 & 32 & 40 & 40 & 30 \\
\hline $\mathrm{K}$ & 77 & M & $\mathrm{L}$ & 41 & 38 & 60 & 64 & 58 \\
\hline $\mathrm{L}$ & 61 & $\mathrm{~F}$ & $\mathrm{~L}$ & 47 & 50 & 56 & 56 & 66 \\
\hline M & 77 & M & $\mathrm{R}$ & 43 & 46 & 36 & 56 & 60 \\
\hline $\mathrm{N}$ & 81 & $\mathrm{~F}$ & $\mathrm{R}$ & 30 & 36 & 44 & 42 & 34 \\
\hline
\end{tabular}

masker level $n_{x}$ was measured at $x=10,15,20$ and $30 \mathrm{~dB}$, and then the $g_{x-\alpha}$ values were measured. The value of $\alpha$ was $5 \mathrm{~dB}$ for $x=10 \mathrm{~dB}$ and $x=15 \mathrm{~dB}$, and $10 \mathrm{~dB}$ for $x=20 \mathrm{~dB}$ and $x=30 \mathrm{~dB}$. The step size of the masker level was $2 \mathrm{~dB}$ for the determination of $n_{x}$, and the step size of $\Delta f / f_{\mathrm{c}}$ was 0.01 for the determination of $g_{x-\alpha}$. The $g_{x-\alpha}$ measurement ranged from 0.0 to 0.6. In the conventional method, the masker level was fixed at $n_{x}$, and then the threshold for detecting the probe was measured. The notch of the masker, $\Delta f / f_{\mathrm{c}}$, was $0.0,0.1,0.2,0.3,0.4$, and 0.6. Initially, these measurements were conducted with the normal-hearing subjects and then the $A_{x-\alpha}$ values in Eqs. (6) and (7) were calculated on the basis of procedure in Fig. 4. It should be noted that when the simplified method is applied to normal-hearing subjects, the values of $g l_{\max }$ and $g u_{\max }$ calculated in Step 5 can be used without calculating acceptable values of $g l_{\max }$ and $g u_{\max }$ using Eqs. (6) and (7) in Step 6. Following the measurements for the normalhearing subjects, the measurements were conducted with the hearing-impaired subjects and then the filter shapes were estimated using the calculated $A_{x-\alpha}$ values.

\subsection{Results}

Table 3 shows the values of $A_{x-\alpha}$ calculated. The $g l_{\max }$ and $g u_{\max }$ values for the hearing impaired subjects were calculated from Eqs. (6) and (7) using $g_{x-\alpha}$ values measured and these $A_{x-\alpha}$ values. The $g l_{\max }$ and $g u_{\max }$

Table 3 Averaged $g_{x-a}$ and $g_{\mathrm{t}}$ for two normal-hearing subjects, and calculated acceptable $g l_{\max }$ and $g u_{\max }$ values from Eqs. (8) and (9), and $A_{x-a}$ values from Eqs. (6) and (7) on the basis of procedure in Fig. 4.

\begin{tabular}{ccccc}
\hline$x \mathrm{~dB}(\alpha \mathrm{dB})$ & $g_{x-a}$ & $g_{\mathrm{t}}$ & $\begin{array}{r}g l_{\max }=g u_{\max } \\
=1.2 g_{\mathrm{t}}\end{array}$ & $\begin{array}{r}A_{x-a}=g l_{\max } / g_{x-a} \\
=g u_{\max } / g_{x-a}\end{array}$ \\
\hline $10(5)$ & 0.111 & 0.29 & 0.35 & 3.15 \\
$15(5)$ & 0.097 & 0.37 & 0.44 & 4.54 \\
$20(10)$ & 0.146 & 0.46 & 0.55 & 3.77 \\
$30(10)$ & 0.156 & 0.58 & 0.70 & 4.49 \\
\hline
\end{tabular}

values calculated were used in the fitting procedure for the simplified method. In this study, these $g l_{\max }$ and $g u_{\max }$ values were also used in the fitting procedure for the conventional method, because $g_{\mathrm{t}}$ values could not be found especially when the auditory filer bandwidth was broadened (i.e., $g_{\mathrm{t}}>0.6$ ). Figure 5(a) shows the correlation diagram of ERB calculated from Eq. (10) obtained using the simplified method and the conventional method for all measurement conditions. ERB data obtained from the simplified method correlates highly with ERB data obtained from the conventional method, in which the correlation is 0.945 . Figure 5(b) shows an example of the auditory filter derived from the both methods. Solid lines denote the simplified method and dashed lines denote the conventional method at $f_{\mathrm{c}}=2,000 \mathrm{~Hz}$ and $x=10 \mathrm{~dB}$ for Subject L. There is no obvious difference between the fitting results for the simplified method and the conventional method. For each auditory filter, the measuring time for $g_{x-\alpha}$ using the simplified method was approximately 2 minutes. The measuring time for $n_{x}$ was approximately 1 minute. The measuring time for the six masked thresholds using the conventional method with the ascending method was approximately 12 minutes.

\section{DISCUSSION}

\subsection{Evaluation of the Ascending Method}

In this simplified method, the hearing threshold $(T)$, the masker level $\left(n_{x}\right)$ and the notch width of the masker $\left(g_{x-\alpha}\right)$ were measured. These are different thresholds in each signal perception task. There may be no obvious difference in the perception task between $T, n_{x}$ and the masked threshold measured using the conventional method, since these three thresholds vary depending on the level of the probe or masker. It is expected, therefore, that $T$ and $n_{x}$ can be measured using the ascending method at a level of accuracy comparable with that of the masked thresholds measured in Experiment I. The perception task of the $g_{x-\alpha}$ is different for these three thresholds, since the threshold 


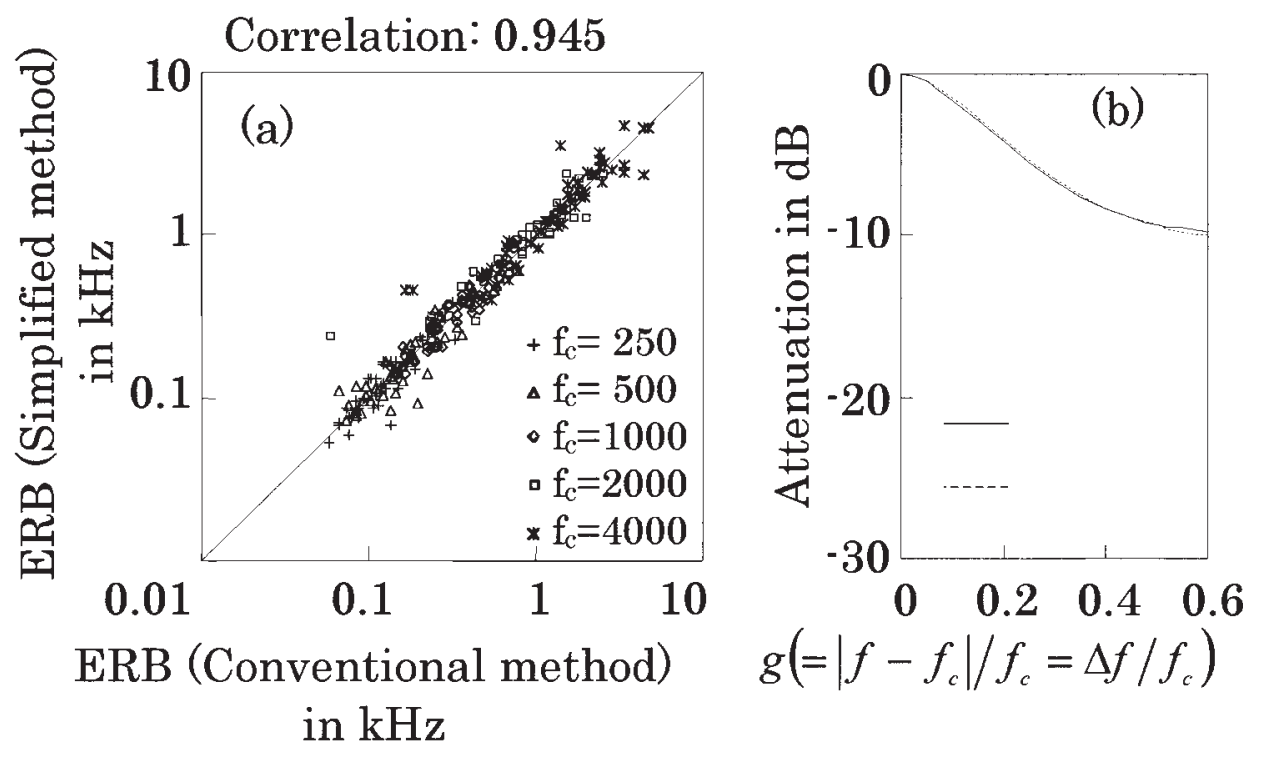

Fig. 5 (a) Correlation between ERB data obtained from the simplified method and the conventional method. The abscissa denotes ERB values using the conventional method and the ordinate denotes ERB values using the simplified measurement method. (b) The estimated auditory filter shapes.

varies depending on the spectral notch width. However, Tyler et al. [23] measured masked thresholds with both varying notch width and varying probe level, and reported that there was no obvious difference between signal perception tasks. Considering Tyler's experimental results, there may be no obvious difference between the ascending method and the 2AFC method in the measurement of $g_{x-\alpha}$. This result suggests that both the conventional method and the simplified method can have sufficient accuracy when using the ascending method.

\subsection{Evaluation of the Simplified Method}

The correlation between ERB obtained from the simplified method and the conventional method is 0.945 . This result shows that it is possible to measure auditory filters using the simplified method at a level of accuracy comparable with that of the conventional method. For the simplified method, the measuring time needed for each auditory filter was approximately 3 minutes (i.e., 2 minutes for the measurement of $g_{x-\alpha}$ and 1 minute for that of $n_{x}$ ), and so the measuring time for the twelve filters described in subsection 2.2.1 of Section 2.2 would be 36 minutes. In addition, the dynamic range of the auditory filter can be established before the measurement by using the determination technique of the masker level, $n_{x}$. This means that an auditory filter located at $x \mathrm{~dB}$ above the hearing threshold can be measured and evaluated. Therefore, it may be possible to evaluate the measured auditory filter quantitatively and intelligibly. By using the white-noise masker with a spectral notch, the auditory filters located in the outside regions of the masker do not affect the measurement of the target filter. Furthermore, the deterioration of measurement accuracy due to an insufficient measurement range of $\Delta f / f_{\mathrm{c}}$ rarely occurs, since the measurement procedure of the simplified method differs from that of the conventional method. Based on these results and considerations, the controversial aspects described in subsections 2.2.1, 2.2.2, 2.2.3 and 2.2.4.1 in Section 2.2 are not present in the simplified method. The determination technique of the $g l_{\max }$ and $g u_{\max }$ values for individual hearing-impaired listeners using parameter $A_{x-\alpha}$ was proposed to remove the controversial aspect 2.2.4.2. As shown in Fig. 5, the simplified method can measure auditory filters to a level of accuracy comparable with that of the conventional method, and thus the controversial aspect 2.2.4.2 may be removed using this technique. This determination technique, however, is based on the assumption of Eqs. (6) through (9), and $B=1.2$. Additionally, in this study, the $A_{x-\alpha}$ values derived from the experimental results for the normalhearing subjects were applied to the estimation procedure for the hearing-impaired subjects. This determination method for $g l_{\max }$ and $g u_{\max }$ should be investigated further from the point of view of both the relationship between $g l_{\max }, g u_{\max }$, and $g_{\mathrm{t}}$, and the availability of $A_{x-\alpha}$ values found in this study.

Taking count of reducing measuring time, the auditory filter shapes are assumed to be symmetric in the simplified method. It is well-known, however, that the shapes are asymmetric. An advanced simplified method is needed to measure the asymmetric auditory filter, in which offfrequency listening [10] should be considered. In the simplified method, additionally, the auditory filter shape is estimated under the condition of $10 \log _{10} r=-x$. Glasberg et al. [18] reported, however, that there are some cases in which the masked thresholds at $\Delta f / f_{\mathrm{c}}$ larger than $g_{\mathrm{t}}$ are higher than the hearing threshold. In these cases, this 
condition $\left(10 \log _{10} r=-x\right)$ may not work well. Measurement and evaluation techniques associated with these cases should be considered in future studies.

In the estimating procedure of the auditory filter shape, correction of the non-flat transmission characteristics of the outer and middle ear is often made. In the simplified method, it may be possible to make the correction. This correction, however, was not made in this study, because the experiments included some subjects with mixed hearing loss, which was a mix of both conductive hearing loss due to disorders of the outer and/or middle ear and cochlear hearing loss.

\section{SUMMARY}

For implementation of this new, usable auditory index, a simplified method has been proposed for the application of auditory filters to the clinical diagnosis of hearing impairment and the fitting of hearing aids. This simplified method is based on the notched-noise method, in which the filter shape is assumed to be symmetric. This method consists of a masker level determination technique for individual hearing-impaired listeners and an auditory filter shape estimation technique using only one measurement point, which corresponds to one masked threshold in the conventional notched-noise method. The measurement accuracy and practicability of this simplified method were investigated experimentally. The results show that (1) signal thresholds can be measured in a short period of time using the ascending method at a level of accuracy comparable with that of the $2 \mathrm{AFC}$ method for auditory filters measurement, (2) the time needed for data collection (i.e., for measuring both the masker level $n_{x}$ and the auditory filter bandwidth $g_{x-\alpha}$ ) is approximately 3 minutes for each auditory filter in the simplified method, and (3) ERB data obtained using the simplified method correlates highly with ERB data obtained using the conventional method. These results show that this simplified method offers sufficient practicability for clinical diagnosis or the fitting of hearing aids. Although this simplified method was developed to measure the auditory filters of hearingimpaired listeners, it is expected that the auditory filters of normal-hearing listeners can also be measured without any problem. In future studies, we also intend to incorporate asymmetry of auditory filters and off-frequency listening into the proposed simplified method.

\section{REFERENCES}

[1] A. E. Carney and D. A. Nelson, "An analysis of psychophysical tuning curves in normal and pathological ears," J. Acoust. Soc. Am., 73, 268-278 (1983).

[2] J. M. Festen and R. Plomp, "Relations between auditory functions in impaired hearing," J. Acoust. Soc. Am., 73, 652662 (1983).

[3] R. S. Tyler, J. W. Hall, B. R. Glasberg, B. C. J. Moore and R.
D. Patterson, "Auditory filter asymmetry in the hearing impaired," J. Acoust. Soc. Am., 76, 1363-1368 (1984).

[4] B. R. Glasberg and B. C. J. Moore, "Auditory filter shapes in subjects with unilateral and bilateral cochlear impairments," $J$. Acoust. Soc. Am., 79, 1020-1033 (1986).

[5] J. R. Dubno and D. D. Dirks, "Auditory filter characteristics and consonant recognition for hearing-impaired listeners," $J$. Acoust. Soc. Am., 85, 1666-1675 (1989).

[6] B. C. J. Moore, D. A. Vickers, B. R. Glasberg and T. Bear, "Comparison of real and simulated hearing impairment in subjects with unilateral and bilateral cochlear hearing loss," Br. J. Audiol., 31, 277-245 (1997).

[7] T. Bear and B. C. J. Moore, "Effects of spectral smearing on the intelligibility of sentences in noise," J. Acoust. Soc. Am., 94, 1229-1241 (1993).

[8] Y. Nejime and B. C. J. Moore, "Simulation of the effect of threshold elevation and loudness recruitment combined with reduced frequency selectivity on the intelligibility of speech in noise," J. Acoust. Soc. Am., 102, 603-615 (1997).

[9] R. D. Patterson, "Auditory filter shapes derived with noise stimuli," J. Acoust. Soc. Am., 59, 640-654 (1976).

[10] R. D. Patterson and I. Nimmo-Smith, "Off-frequency listening and auditory-filter asymmetry," J. Acoust. Soc. Am., 67, 229245 (1980)

[11] B. C. J. Moore and B. R. Glasberg, "Formulae describing frequency selectivity as a function of frequency and level, and their use in calculating excitation patterns," Hear. Res., 28, 209-225 (1987).

[12] B. R. Glasberg, B. C. J. Moore, R. D. Patterson and I. NimmoSmith, "Dynamic range and asymmetry of the auditory filter," J. Acoust. Soc. Am., 76, 419-427 (1984).

[13] S. Rosen, R. J. Baker and A. Darling, "Auditory filter nonlinearity at $2 \mathrm{kHz}$ in normal hearing listeners," J. Acoust. Soc. Am., 103, 2539-2550 (1998).

[14] H. Fletcher, "Auditory patterns," Rev. Mod. Phys., 12, 47-65 (1940).

[15] R. D. Patterson, I. Nimmo-Smith, D. L. Weber and R. Milroy, "The deterioration of hearing with age: Frequency selectivity, the critical ratio, the audiogram, and speech threshold," $J$. Acoust. Soc. Am., 72, 1788-1803 (1982).

[16] B. R. Glasberg and B. C. J. Moore, "Derivation of auditory filter shapes from notched-noise data," Hear. Res., 47, 103138 (1990).

[17] B. C. J. Moore, B. R. Glasberg and T. Bear, "A model for perception of thresholds, loudness and partial loudness," $J$. Audio Eng. Soc., 45, 224-240 (1997).

[18] B. R. Glasberg and B. C. J. Moore, "Frequency selectivity as a function of level and frequency measured with uniformly exciting notched noise," J. Acoust. Soc. Am., 108, 2318-2328 (2000).

[19] H. Levitt, "Transformed Up-down methods in psychoacoustics," J. Acoust. Soc. Am., 49, 467-477 (1971).

[20] M. A. Stone, B. R. Glasberg and B. C. Moore, "Simplified measurement of auditory filter shapes using the notched-noise method," Br. J. Audiol., 26, 329-334 (1992).

[21] M. J. Shailer, B. C. J. Moore, B. R. Glasberg, N. Watson and S. Harris, "Auditory filter shapes at 8 and $10 \mathrm{kHz}, "$ J. Acoust. Soc. Am., 88, 141-148 (1990).

[22] W. H. Press, B. P. Flannery, S. A. Teukolsky and W. T. Vetterling, Numerical Recipes in $C$ (Cambridge University press, Cambridge, 1988).

[23] R. S. Tyler and N. Tye-Murray, "Frequency resolution measured by adaptively varying the notch width: Results from normals and hearing impaired," in Auditory Frequency Selectivity, B. C. J. Moore, R. D. Patterson, Eds. (Kluwer Academic / Plenum Publishers, Dordrecht, 1986), pp. 323330. 


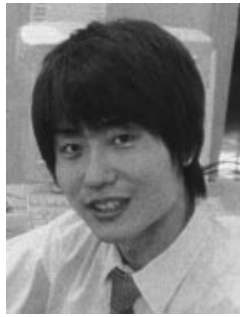

Takeshi Nakaichi graduated from Tokyo Denki University in 1995. He is currently a researcher and an engineer in RION Co., Ltd. He engages in research on new auditory index for hearing impairments and new auditory prosthesis techniques. He is a member of the Acoustical Society of Japan.

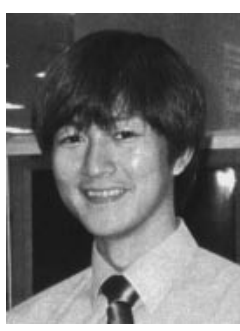

Keisuke Watanuki received the M.E. degrees from Musashi Institute of Technology in 1997. $\mathrm{He}$ is currently a researcher and an engineer in RION Co., Ltd. His research interests include simulation of auditory characteristics of hearing impairments and development of new auditory prosthesis techniques. $\mathrm{He}$ is a member of the Acoustical Society of Japan.

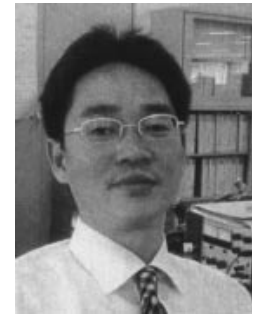

Shinichi Sakamoto received the M.E. degree from Kogakuin University in 1991. He is currently a researcher in RION Co., Ltd. His research interests include auditory characteristics of hearing impaired people and developing new auditory prosthesis techniques. He is a member of the Acoustical Society of Japan, the Institute of Electronics, Information and Communication Engineers, Audiology Japan and the Institute of Noise Control Engineering Japan. 